\title{
NDVI COMPUTATION OF LISS III IMAGES USING QGIS
}

\author{
Vijayalakshmi V ${ }^{1}$, D Mahesh Kumar², S C Prasanna Kumar ${ }^{3}$ and Thejaswini P. ${ }^{4}$ \\ ${ }^{1}$ Research Scholar, J S S Academy of Technical Education, Bengaluru,v.vijayalakshmi80@gmail.com, \\ ${ }^{2,4}$ J S S Academy of Technical Education, Bengaluru, ${ }^{2}$ dmkjssate@gmail.com, ${ }^{4}$ thejaswini@jssateb.ac.in. \\ ${ }^{3} R$ V College of Engineering, Bengaluru,prasannakumar@rvce.edu.in,
}

\begin{abstract}
Feature Selection and Extraction is a very significant and mandatory part in the domain of image processing. After the relevant preprocessing operations, the relevant features have to be extracted using suitable algorithms. In multispectral imagery, the features are identified and extracted based on the applications and objectives of the analysis such as color, texture, brightness, intensity etc. Some of the prominent algorithms used for feature extraction are mean shift algorithm, Principal Component transformation, Wavelet based Transformation, Local Binary Patterns etc. Texture based feature detection and extraction is the most prominent method adopted which involves multispectral images. With respect to hyperspectral images, dimensionality is a critical issue to be dealt appropriately.
\end{abstract}

Keywords-Feature detection and Extraction, Satellite imagery, textures,

\section{INTRODUCTION}

Feature detection and extraction is a mandatory phase in information extraction and classification of multispectral and hyperspectral images as it filters out the undesired part of the images and also reduces the dimension of the input image. Feature extraction involves mapping of input pixels into a subspace of reduced dimensionality with respect to the original feature space using either linear or nonlinear approaches. The process of feature extraction not only generates a sub-image of the input image but also reduces the computation power and memory requirements of the CPU. Optimal features are charactarised by class separability .The processing of multispectral and hyperspectral images differs with the fact that the size of training data being proportional to the dimensions of the image. Image transformation based on textures modifies the image based on the spatial properties of the pixel.

In texture based segmentation technique, pixels are ordered based on homogeneous textures.

\section{LITERATURE REVIEW}

Automatic feature detection and extraction technique using multispectral images is the contribution of this paper.. Here Delaunay graph and the Voronoi tessellation are employed for feature detection and extraction. Image segmentation aids in detecting of edges which are composed of high intensity pixels. Objects are chosen based on color property of the pixel. Using binary subtraction operation, the original image is subtracted with its morphologically dilated or eroded image. Since the segmentation process might lead to misclassification resulting in image quality deterioration, appropriate image preprocessing operations needs to be performed[1].

A texture unit, comprising of two separable units with four elements were represented which were formed from a neighborhood of eight elements. Each of these units were called cross texture and diagonal texture units. With reference to every pixel, a Cross Diagonal Texture Matrix was generated using different combinations of cross and diagonal texture units which was shown to be time efficient. It was shown that median technique also aided in noise removal in satellite images. Structural and statistical are two approaches used in texture analysis[2].

A wavelet based transformation method has been adopted to resolve the dimensionality issue that occurs with respect to hyperspectral images. Principal Component Transformation approach has been used whose vectors are generated based on Karhunen-Loéve's method. For optimal Class Separability, Linear Discriminant Analysis approach has been adopted. The need for exponential rise in the number of training samples is avoided as feature extraction based on wavelet transforms optimizes in selecting effective features. Supervised classification has been performed on the features which were extracted. For bast signal approximation, a Matching Pursuit is used with a greedy strategy. The paper concludes by stating that appropriate schemes have to be used for accuracy assessment[3].

World-View II images of the study area Dongsha Atoll and Landsat images of the study area Zengmu shoal were taken for study. Principal Component Analysis was performed on the World-View images whose resultant first component image consisted of maximum information. The Level Set method is used as a segmentation scheme which uses the number of objects and the boundary extracted as a metrics for analysis. The Level Set method uses on isocontour curve which is dependent on the energy of the image serves as a baseline value. It is observed that the initial curve is moving towards an area of minimum energy within the image and thus the area boundary is generated. It was observed that the number of segments within a band was proportional to the information content. An area of 100 sq $\mathrm{m}$ was set a threshold to count the number of images and 
which also supported in noise removal. Normalized Differential Water Index(NDWI) was evaluated using the coastal aerosol and NIR2 bands[4].

Fourier Transform based texture analysis approach has been adopted in this paper to discriminate different types of forests and to perform soil studies using images with less reflectance and contrast values. A co-occurrence matrix generated represents the spatial distribution of pixel gray levels[5].

Extracting and classifying, both spatial and spectral features with optimal accuracy, using advanced techniques such as Deep Belief Networks(DBN) on multispectral images is implemented in this paper. The study area considered is Kottayam, India and images of LISS-III satellite are chosen. Land Use Land Cover Classification(LULC) of the study area has been done using DBN, with unsupervised learning approach. DBNs are composed of two layers Restricted Boltzmann Machine (RBM) layer and Logical Regression(LR) layers with an architecture similar to that of Artificial Neural Networks(ANN). Local Binary Pattern(LBP) approach has been implemented as a tool for extracting features. It was observed that using DBN approach resulted in classification of higher accuracy than compared to k-means clustering, cmeans clustering in combination with LBP and Support Vector Machine(SVM) based LBP method. Finally Kappa Co-efficient based accuracy assessment was performed to compare the performances of all the three methods[6].

An Normalized Differential Vegetation Index(NDVI) based approach has been used to generate the spectral signature of the objects for the purpose of Land Cover Classification. The study area selected is Jabalpur, India. Landsat-7 Images consisting of 7 bands of the study area were used in NDVI analysis to detect the presence of vegetation. NDVI was computed using the combinations of Red band with NIR and Green with NIR(Near Infra Red). The paper concludes by extending threshold based NDVI analysis to various other applications such as natural disaster management, agricultural based soil studies, crop pattern analysis etc[7].

A combination of Gray Level Co-occurrence (GLCM) and Lifting Wavlet(LWT) approach has been implemented for feature extraction. Further the feature vector is trained using SVM for object identification. Implementing a combination of median filter with Contrast Limited Adaptive Histogram Equalization(CLAHE) in the preprocessing phase helps in retaining the edges and denoising the input image. Some common first order features along with GLCM and LWT features were extracted to generate the feature vector. SVM can be used in the classification of soil and other land cover classes[8].

Textural properties are extracted using GLCM composing of second order statistical parameters. A combination of MATLAB with Verilog which is a type of Hardware Description Language was used in this work. Conversion of the input preprocessed image into a hex file was performed to be further processed by the Verilog Simulation Software. LISS-IV image of the study area i.e Madurai, India was taken for implementation. A novel approach using Field Programmable Gate Array(FPGA) has been implemented in this work to efficiently and effectively handle the enormity of the remotely sensed imaged in terms of memory and computation power. The paper concludes by stating that real time sequential fetching of date needs to be done in the future[9].

An Artificial Neural Network(ANN) based land cover classification approach has been found to be very effective in optimizing accuracy. Multi spectral images of the Bairelli city, India has been procured from Google Earth. The Levenberg-Marquardt algorithm was designed to approach second-order training speed. The Jacobian matrix can be computed through a standard backpropagation technique. The paper concludes by stating that a multisource , multimodal dataset would yield better classification results[10].

\section{STUDY AREA AND DATA}

The study area considered in this approach is that of parts of Mysore district located at the south Karnataka state, India. Indian Remote Sensing(IRS)-LISS III (Linear Imaging Self Scanning) multispectral images of the study area were downloaded to perform computation of NDVI for analyzing growth of vegetation. LISS III images have a resolution of $24 \mathrm{~m}$, with a swath of $140 \mathrm{~km}$ and revisit period of 24 days. These images consist of four spectral bands covering the wavelength as shown in the table.

TABLE I. SPECTRAL BANDS OF LISS III IMAGES.

\begin{tabular}{|l|l|c|}
\hline $\begin{array}{c}\text { S1 } \\
\text { No }\end{array}$ & \multicolumn{1}{|c|}{ Spectral Band } & $\begin{array}{c}\text { Wavelength } \\
\text { range in } \boldsymbol{\mu m}\end{array}$ \\
\hline 1 & Band 2- Green & $0.52-0.59$ \\
\hline 2 & Band 3- Red & $0.62-0.68$ \\
\hline 3 & Band 4- Near Infra Red(NIR) & $0.77-0.86$ \\
\hline 4 & $\begin{array}{l}\text { Band5- Short Wave Infra } \\
\text { Red(SWIR) }\end{array}$ & $1.55-1.7$ \\
\hline
\end{tabular}

\section{Methodology}

Computation of NDVI using an interpreter language such as PYTHON provides flexibility in coding. This paper makes an attempt to process satellite images using Jupyter Notebook. Band 3(Red) and Band 4(NIR) of LISS III image was imported using rasterio and matplotlib python libraries.

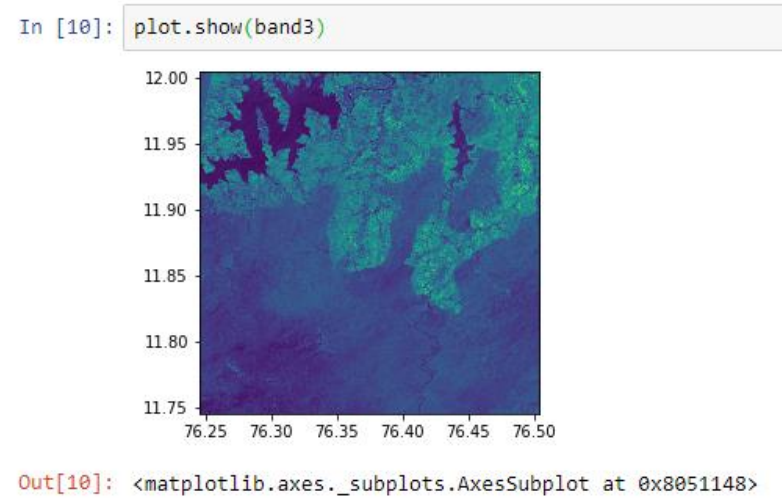

Fig. 1. Band 3 (Red) of LISS III 


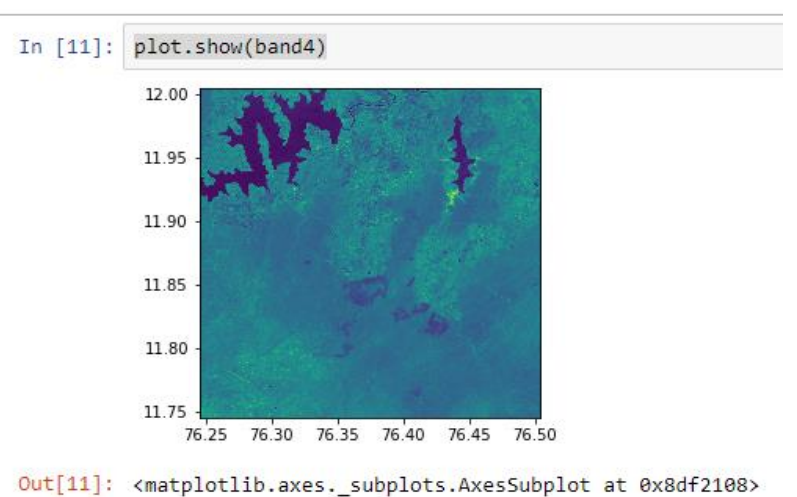

Fig. 2. Band 4 (NIR) of LISS III image

The array representation of both the images were obtained using the read function.

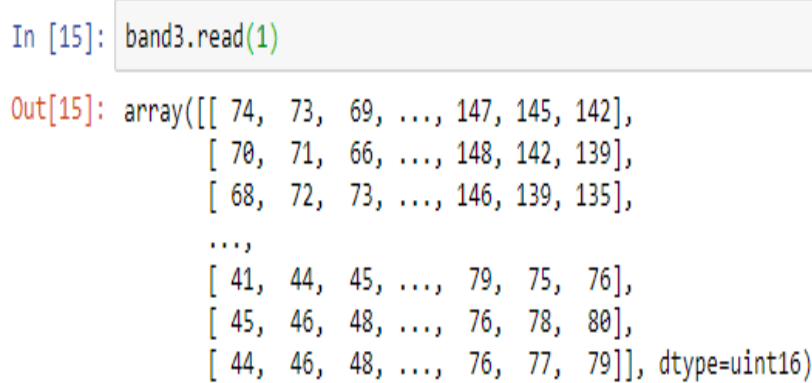

Fig. 3. Band 3(Red) Array Representation

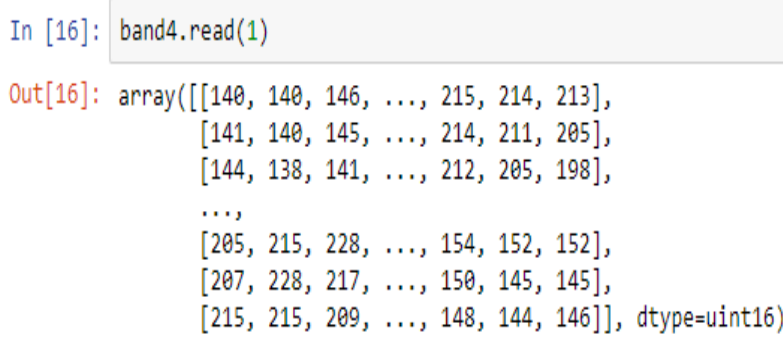

Fig. 4. Band 4 (NIR) Array Represenattion

The height and width of the image was found to be 1151 with EPSG:4326 as the co-ordinate reference system. The unsigned int datatype was then converted to float64 datatype.

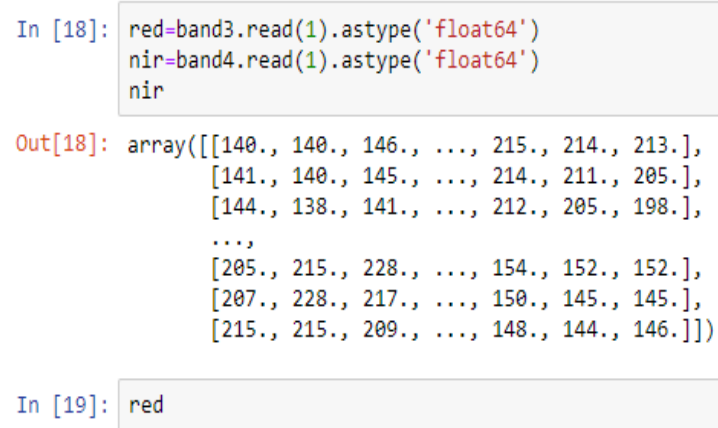

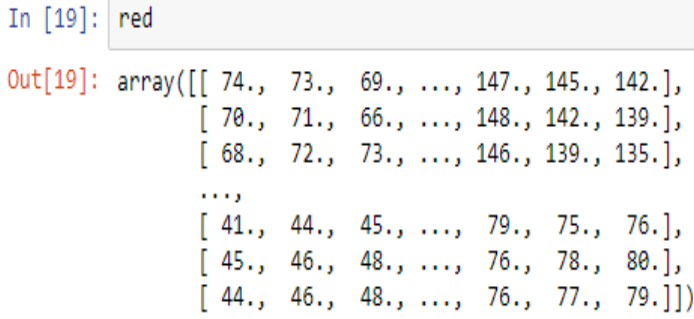

Fig. 5. Band 4 (NIR) and Band 3(Red) Float converted Array Represenattions

NDVI was computed using equation (1).

$$
N D V I=(N I R-R E D) /(N I R+R E D)
$$

The array representation of the computed values was obtained as follows:

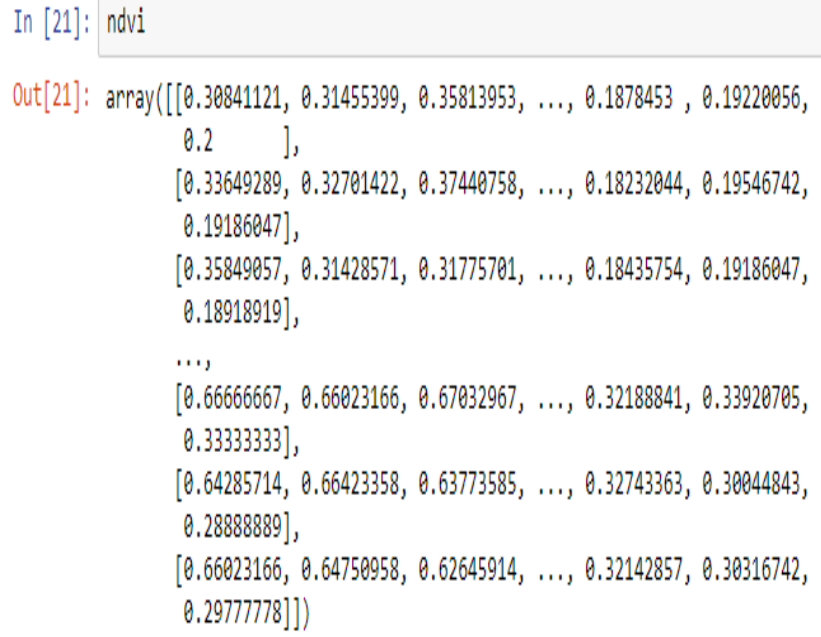

Fig. 6. Array Represenattion of NDVI values.

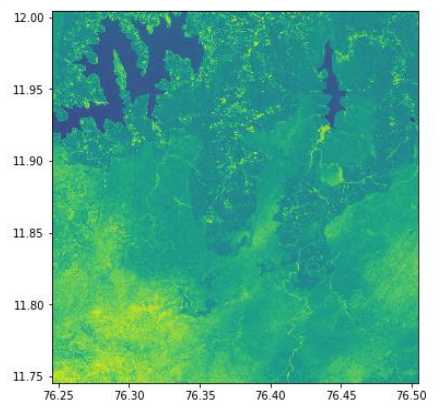

Fig. 7. NVDI Image

Fig. 7. Shows the NDVI image output representation of the study area. This image will be further classified into various classes based on the calculated NDVI values using a suiatble GIS platform. In this work Quantum Geographic Information System(QGIS) was used as it is an open source. Hence the NDVI image was imorted into QGIS platform by adding it as a raster layer. Fig. 8. Shows the raster layer added into QGIS with only two values i.e black and white. 


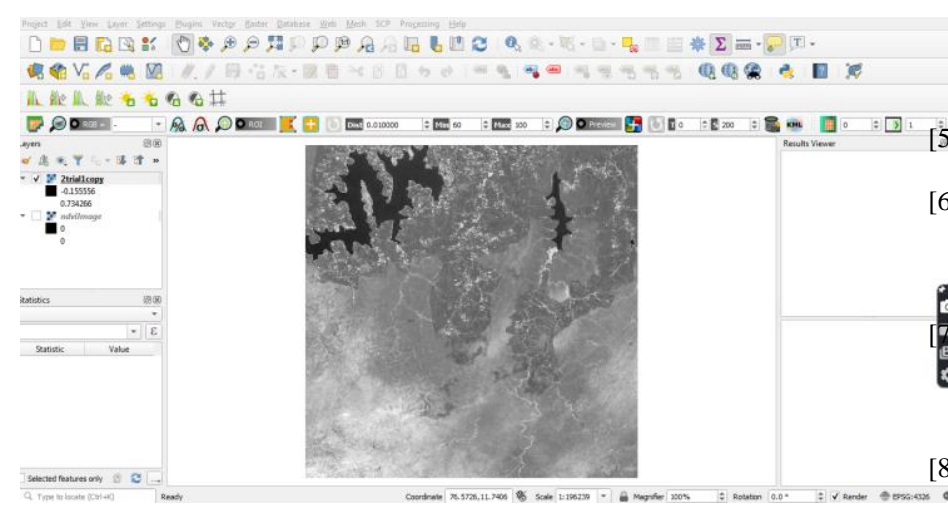

Fig. 8. NDVI image in QGIS

\section{RESUlTS AND DisCUSSIONS}

The NDVI image is further classified into five classes based upon the computed NDVI values in QGIS platform. The values were found to be in the range of 0.73 to -0.15 . The classification was performed by selecting equal intervals for all the five classes. A linear interpolation technique was used in this approach. Fig. 9. Shows the classification of NDVI image into five classes.

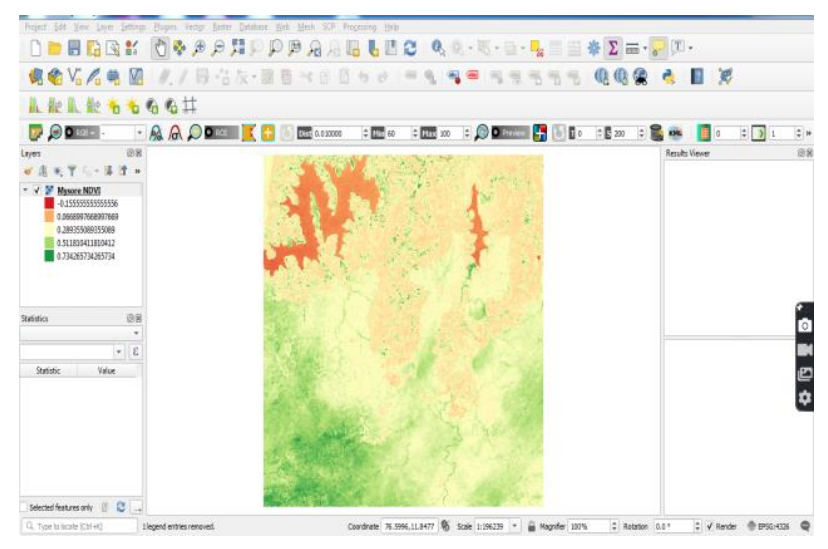

Fig. 9. Classified NDVI image using QGIS.

It was found that a value of $0.73,0.51,0.28,0.66$ and -0.15 corresponded very high, high, moderate, sparse and no vegetation levels respectively. Using the Feature identification tool on QGIS, relevant features can be identified. Features can be detected and extracted for application specific domains.

\section{REFERENCES}

[1] Sharma, Ojaswa; Mioc, Darka; Anton, François, "Polygon Feature Extraction from Satellite Imagery Based on Colour Image Segmentation and Medial Axis," 2008.

[2] Amit K Bhattacharya, P K Srivastava, Anil Bhagat, “ A Modified Texture Filtering Technique for Satellite Images," $22^{\text {nd }}$ Asian Conference on Remote Sensing, Nov 2001, Singapore.

[3] P H hsu, "Feature Extraction of Hyperspectral Images for matching Pursuit," National Science and Technology for Disaster Reduction.

[4] Peter Tian-Yuan Shih, Jian-Wei Lin, Wei-Tsun Lin, and Cheng-Gi Wang, "Underwater Linear Feature Extraction With Multispectral
Band Images:

An Evaluation With Level-Set Method In Dongsha

Atoll And Zengmu Shoal", Journal of Marine Science and

Technology, Vol. 24, No. 6, pp. 1226-1233,2016.

[5] Robert Riou and Frederique Seyler, "Texture Analysis of Tropical Rain Forest Infrared Satellite Images."

[6] T Vighnesh, K K Thyagharajan, "Land Use and Land Cover Classification Using Deep Belief Network for LISS-III Multispectral Satellite Images," International Journal of Innovative Technology and Exploring Engineering, Volume-9 Issue-1S, November 2019.

A. K. Bhandari , A. Kumar, and G. K. Singh, "Feature Extraction using Normalized Difference Vegetation Index (NDVI): a Case Study of Jabalpur City," 2nd International Conference on Communication, Computing \& Security [ICCCS-2012].

[8] Anita Dixit, Dr. Nagaratna Hedge and Dr. B. Eswar Reddy, “ Texture Feature Based Satellite Image Classification Scheme Using SVM," International Journal of Applied Engineering Research ISSN 09734562 Volume 12, Number 13 (2017).

[9] P Steffi Shakila, Dr.V Vinoth Thyagarajan ,Dr.S.Rajaram, “ Texture Feature Extraction for Multispectral Satellite Image using Verilog HDL," International Research Journal of Engineering and Technology Volume: 05, Issue: 05 , May-2018.

[10] Madhavi Agnihotri, Sanjay Srivastava, "Feature Extraction of a Multispectral Image by a Neural Approach," International Journal of Advanced Research in Computer Science \& Technology, Vol. 3, Issue 1 (Jan. - Mar. 2015). 